\title{
Piecewise-linear models of genetic regulatory networks: theory and example
}

\author{
Frédéric Grognard ${ }^{1}$, Hidde de Jong ${ }^{2}$, and Jean-Luc Gouzé ${ }^{1}$ \\ 1 COMORE INRIA, Unité de recherche Sophia Antipolis, 2004 route des Lucioles, BP 93, \\ 06902 Sophia Antipolis, France.frederic.grognardeinria.fr, \\ gouzedsophia.inria.fr \\ 2 HELIX INRIA, Unité de recherche Rhône-Alpes, 655 avenue de l'Europe, Montbonnot, \\ 38334 Saint Ismier Cedex, France.Hidde.de-Jongeinrialpes. fr
}

Summary. The experimental study of genetic regulatory networks has made tremendous progress in recent years resulting in a huge amount of data on the molecular interactions in model organisms. It is therefore not possible anymore to intuitively understand how the genes and interactions together influence the behavior of the system. In order to answer such questions, a rigorous modeling and analysis approach is necessary. In this chapter, we present a family of such models and analysis methods enabling us to better understand the dynamics of genetic regulatory networks. We apply such methods to the network that underlies the nutritional stress response of the bacterium $E$. coli.

The functioning and development of living organisms is controlled by large and complex networks of genes, proteins, small molecules, and their interactions, socalled genetic regulatory networks. The study of these networks has recently taken a qualitative leap through the use of modern genomic techniques that allow for the simultaneous measurement of the expression levels of all genes of an organism. This has resulted in an ever growing description of the interactions in the studied genetic regulatory networks. However, it is necessary to go beyond the simple description of the interactions in order to understand the behavior of these networks and their relation with the actual functioning of the organism. Since the networks under study are usually very large, an intuitive approach for their understanding is out of question. In order to support this work, mathematical and computer tools are necessary: the unambiguous description of the phenomena that mathematical models provide allows for a detailed analysis of the behaviors at play, though they might not exactly represent the exact behavior of the networks.

In this chapter, we will be mostly interested in the modeling of the genetic regulatory networks by means of differential equations. This classical approach allows precise numerical predictions of deterministic dynamic properties of genetic regulatory networks to be made. However, for most networks of biological interest the application of differential equations is far from straightforward. First, the biochemical reaction mechanisms underlying the interactions are usually not or incompletely 
known, which complicates the formulation of the models. Second, quantitative data on kinetic parameters and molecular concentrations is generally absent, even for extensively-studied systems, which makes standard numerical methods difficult to apply. In practice, the modeler disposes of much weaker information on the network components and their interactions. Instead of details on the mechanisms through which a protein regulates a gene, we typically only know whether the protein is an activator or an inhibitor. And even if it had been shown, for example, that the protein binds to one or several sites upstream of the coding region of the gene, numerical values of dissociation constants and other parameters are rarely available. At best, it is possible to infer that the regulatory protein strongly or weakly binds to the DNA, with a greater affinity for one site than for another.

Due to those uncertainties, we cannot hope to build a model that is guaranteed to reproduce the exact behavior of the considered genetic regulatory network. No model will be quantitatively accurate. It is therefore necessary to concentrate on the construction of models that reproduce the qualitative dynamical properties of the network, that is, dynamical properties that are invariant for a range of parameter values and reaction mechanisms. The qualitative properties express the intimate connection between the behavior of the system and the structure of the network of molecular interactions, independently from the quantitative details of the latter.

Consequently, qualitative approaches have been developed for the modeling, analysis, and simulation of genetic regulatory networks and other networks of biological interactions: Boolean networks [20,30], Petri nets [22, 27], process algebras [28], qualitative differential equations [17], hybrid automata [11],... In this chapter, we concentrate on one particular class of qualitative models of genetic regulatory networks, originally proposed by Glass and Kauffman [12]: piecewise-linear (PL) differential equations. In Section 1, we describe this family of models and give a small example. In Section 2, we show qualitative results that have been obtained for the analysis of such systems. We then illustrate these models on the nutritional stress response of E. coli in Section 3, before discussing remaining challenges for the analysis and control of such models in Section 4.

\section{Models of genetic regulatory networks}

Among the many emerging families of models (see [5]), a class of piecewise-linear (PL) models, originally proposed by Glass and Kauffman [12], has been widely used in modeling genetic regulatory networks. The variables in the piecewise-linear differential equation (PLDE) models are the concentrations of proteins encoded by the genes, while the differential equations describe the regulatory interactions in the network by means of step functions. The use of step functions is motivated by the switch-like behavior of many of the interactions in genetic regulatory networks [26], but it leads to some mathematical difficulties. The vector field for the PLDE model is undefined when one of the variables assumes a value where the step function is discontinuous, referred to as a threshold value. Recent work by Gouzé and Sari [13] uses an approach due to Filippov to define the solutions on the threshold hyperplanes. 
The approach involves extending the PLDE to a piecewise-linear differential inclusion (PLDI). As is well known, such discontinuities can lead to sliding modes. The definitions and results of this section are mainly taken from [3].

The family of PL-models is best illustrated with an example: the schematic diagram in Figure 1 describes a simple genetic regulatory network. In this example, the genes $a$ and $b$ code for the proteins $\mathrm{A}$ and $\mathrm{B}$, which in turn control the expression of the two genes $a$ and $b$. Protein A inhibits gene $a$ and activates gene $b$ above certain threshold concentrations, which are assumed to be different. Similarly protein B inhibits gene $b$ and activates gene $a$ above different threshold concentrations. This two-gene regulatory network is simple but represents many features of regulation found in real networks: auto-regulation, cross-regulation and inhibition/activation. Such a two-gene network could be found as a module of a more complex genetic regulatory network from a real biological system.

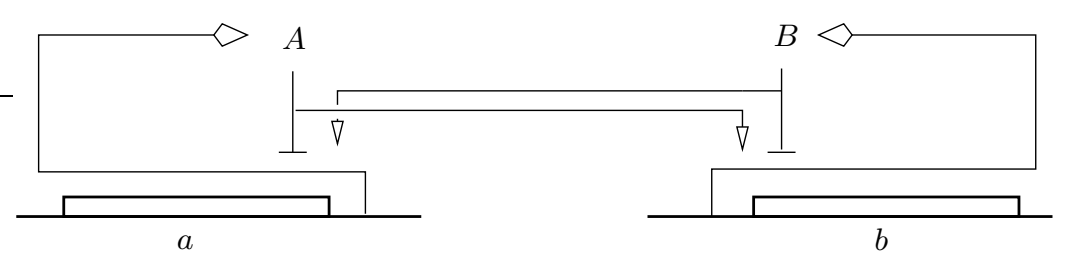

Fig. 1. Example of a genetic regulatory network of two genes ( $a$ and $b$ ), each coding for a regulatory protein (A and $\mathrm{B})$.

The equations modeling the example network in Figure 1 can be written down as

$$
\left\{\begin{array}{l}
\dot{x}_{a}=\kappa_{a} s^{+}\left(x_{b}, \theta_{b}^{1}\right) s^{-}\left(x_{a}, \theta_{a}^{2}\right)-\gamma_{a} x_{a} \\
\dot{x}_{b}=\kappa_{b} s^{+}\left(x_{a}, \theta_{a}^{1}\right) s^{-}\left(x_{b}, \theta_{b}^{2}\right)-\gamma_{b} x_{b}
\end{array}\right.
$$

where $s^{+}\left(x_{s}, \theta_{s}\right)$ is equal to 0 when $x_{s}<\theta_{s}$ and equal to 1 when $x_{s}>\theta_{s}$ and $s^{-}\left(x_{s}, \theta_{s}\right)=1-s^{+}\left(x_{s}, \theta_{s}\right)$. In this model, gene $a$ is expressed at a rate $\kappa_{a}$ if the concentration $x_{b}$ of protein $b$ is above the threshold $\theta_{b}^{1}$ and the concentration $x_{a}$ of protein A is below the threshold $\theta_{a}^{2}$. Similarly, gene $b$ is expressed at a rate $\kappa_{b}$ if the concentration $x_{a}$ of protein $\mathrm{A}$ is above the threshold $\theta_{a}^{1}$ and the concentration $x_{b}$ of the protein $\mathrm{B}$ is below the threshold $\theta_{b}^{2}$. Degradation of both proteins is assumed to be proportional to their own concentrations, so that the expression of the genes $a$ and $b$ is modulated by the degradation rates $\gamma_{a} x_{a}$ and $\gamma_{b} x_{b}$ respectively.

Such a model is readily generalized to models containing both expression and degradation terms for each gene:

$$
\dot{x}_{i}=f_{i}(x)-\gamma_{i} x_{i}
$$

where $f_{i}(x)$ represents the expression rate of gene $i$, depending on the whole state $x=\left(x_{1}, \cdots, x_{n}\right)^{T}$ and $\gamma_{i} x_{i}$ is the degradation rate. However, the expression rates of (1) have the additional property of being constant for values of $x_{a}$ and $x_{b}$ belonging 
to intervals that do not contain thresholds values $\theta_{i}^{j}$. This can be rewritten by detailing $f_{i}(x)$ as follows:

$$
f_{i}(x)=\sum_{l=1}^{L_{i}} \kappa_{i l} b_{i l}(x)
$$

where $b_{i l}(x)$ is a combination of step-functions $s^{ \pm}\left(x_{r}, \theta_{r}^{j}\right)$ and $\kappa_{i l}>0$ is a rate parameter. The generalized form of (1) is a piecewise linear model

$$
\dot{x}=f(x)-\gamma x
$$

where the model is linear within hyper-rectangles of the state-space.

The dynamics of the piecewise-linear system (2) can be studied in the $n$ dimensional state-space $\Omega=\Omega_{1} \times \Omega_{2} \times \cdots \times \Omega_{n}$, where each $\Omega_{i}$ is defined by $\Omega_{i}=\left\{x \in \mathbb{R}_{+} \mid 0 \leq x_{i} \leq \max _{i}\right\}$ for some positive parameter $\max _{i}>$ $\max _{x \in \Omega}\left(\frac{f_{i}(x)}{\gamma_{i}}\right)$. A protein encoded by a gene will be involved in different interactions at different concentration thresholds, so for each variable $x_{i}$, we assume there are $p_{i}$ ordered thresholds $\theta_{i}^{1}, \cdots, \theta_{i}^{p_{i}}$ (we also define $\theta_{i}^{0}=0$ and $\theta_{i}^{p_{i}+1}=\max _{i}$ ). The $(n-1)$-dimensional hyperplanes defined by these thresholds partition $\Omega$ into hyper-rectangular regions we call domains. Specifically, a domain $D \subset \Omega$ is defined to be a set $D=D_{1} \times \cdots \times D_{n}$, where $D_{i}$ is one of the following:

$$
\begin{aligned}
& D_{i}=\left\{x_{i} \in \Omega_{i} \mid 0 \leq x_{i}<\theta_{i}^{1}\right\} \\
& D_{i}=\left\{x_{i} \in \Omega_{i} \mid \theta_{i}^{j}<x_{i}<\theta_{i}^{j+1}\right\} \\
& D_{i}=\left\{x_{i} \in \Omega_{i} \mid \theta_{i}^{p_{i}}<x_{i} \leq \max _{i}\right\} \\
& D_{i}=\left\{x_{i} \in \Omega_{i} \mid x_{i}=\theta_{i}^{j}\right\} \quad \text { for } j \in\left\{1, \cdots, p_{i}-1\right\}
\end{aligned}
$$

A domain $D \in \mathcal{D}$ is called a regulatory domain if none of the variables $x_{i}$ has a threshold value in $D$. In contrast, a domain $D \in \mathcal{D}$ is called a switching domain of order $k \leq n$ if exactly $k$ variables have threshold values in $D$ [25]. The corresponding variables $x_{i}$ are called switching variables in $D$. For convenience, we denote the sets of regulatory and switching domains by $\mathcal{D}_{r}$ and $\mathcal{D}_{s}$ respectively. It is also useful to define the concept of a supporting hyperplane for a domain.

Definition 1. For every domain $D \in \mathcal{D}_{s}$ of order $k \geq 1$, define supp $(D)$ to be the $(n-k)$-dimensional hyperplane containing $D$. If $D \in \mathcal{D}_{r}$ then we define supp $(D)$ to be equal to $\Omega$.

\subsection{Solutions in regulatory domains}

For any regulatory domain $D \in \mathcal{D}_{r}$, the function $f(x)$ is constant for all $x \in D$, and it follows that the piecewise-linear system (2) can be written as a linear vector field

$$
\dot{x}=f^{D}-\gamma x
$$

where $f^{D}$ is constant in $D$. Restricted to $D$, this is a classical linear ordinary differential equation. From (3), it is clear that all solutions in $D$ monotonically converge 
towards the corresponding equilibrium $\phi(D)$, which is defined by $\gamma \phi(D)=f^{D}$. If $\phi(D)$ belongs to the closure of $D$, all solutions initiated in $D$ converge towards $\phi(D)$; otherwise, all solutions reach the boundary of $D$ in finite time (which means that they exit $D$ ).

Definition 2. Given a regulatory domain $D \in \mathcal{D}_{r}$, the point $\phi(D)=\gamma^{-1} f^{D} \in \Omega$ is called the focal point for the flow in D.

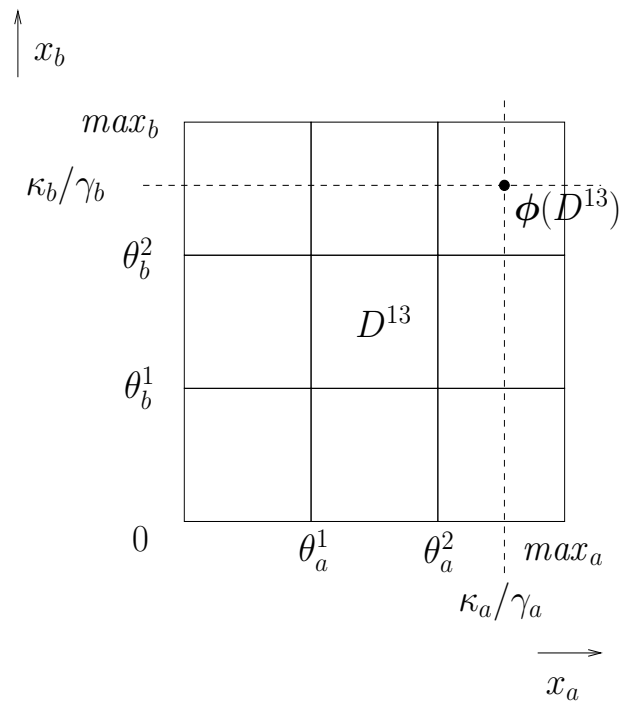

Fig. 2. Illustration of the focal point $\phi\left(D^{13}\right)$ of a domain $D^{13}$ in example (1)

In Figure 2, example (1) is used to illustrate this concept: the considered regulatory domain $D$ is $\left\{x_{a} \in \Omega_{a} \mid \theta_{a}^{1}<x_{a}<\theta_{a}^{2}\right\} \times\left\{x_{b} \in \Omega_{b} \mid \theta_{b}^{1}<x_{b}<\theta_{b}^{2}\right\}$, so that system (1) becomes

$$
\left\{\begin{array}{l}
\dot{x}_{a}=\kappa_{a}-\gamma_{a} x_{a} \\
\dot{x}_{b}=\kappa_{b}-\gamma_{b} x_{b}
\end{array}\right.
$$

and the corresponding focal point is $\left(\frac{\kappa_{a}}{\gamma_{a}}, \frac{\kappa_{b}}{\gamma_{b}}\right)$. In the figure, this focal point is supposed to be outside of $D$ : every solution starting in $D$ therefore exits this domain in finite time.

\subsection{Solutions in switching domains}

In switching domains, the PL system (2) is not defined, since in a switching domain of order $k \geq 1, k$ variables assume a threshold value. If solutions do not simply go through a switching domain, it is necessary to give a definition of what a solution 
can be on that domain. Classically, this is done by using a construction originally proposed by Filippov [10] and recently applied to PL systems of this form [13, 7].

The method consists of extending the system (3) to a differential inclusion,

$$
\dot{x} \in H(x),
$$

where $H$ is a set-valued function (i.e. $H(x) \subseteq \mathbb{R}^{n}$ ). If $D$ is a regulatory domain, then we define $H$ simply as

$$
H(x)=\left\{f^{D}-\gamma x\right\},
$$

for $x \in D$. If $D$ is a switching domain, for $x \in D$, we define $H(x)$ as

$$
H(x)=\overline{c o}\left(\left\{f^{D^{\prime}}-\gamma x \mid D^{\prime} \in R(D)\right\}\right),
$$

where $R(D)=\left\{D^{\prime} \in \mathcal{D}_{r} \mid D \subseteq \partial D^{\prime}\right\}$ is the set of all regulatory domains with $D$ in their boundary, and $\overline{c o}(X)$ is the closed convex hull of $X$. For switching domains, $H(x)$ is generally multi-valued so we define solutions of the differential inclusion as follows.

Definition 3. A solution of (4) on $[0, T]$ in the sense of Filippov is an absolutely continuous function (w.r.t. $t) \xi_{t}\left(x_{0}\right)$ such that $\xi_{0}\left(x_{0}\right)=x_{0}$ and $\dot{\xi}_{t} \in H\left(\xi_{t}\right)$, for almost all $t \in[0, T]$.

In order to more easily define these Filippov solutions, it is useful to define a concept analogous to the focal points defined for regulatory domains, extended to deal with switching domains.

Definition 4. Let $D \in \mathcal{D}_{s}$ be a switching domain of order $k$. Then its focal set $\Phi(D)$ is

$$
\Phi(D)=\operatorname{supp}(D) \cap \overline{c o}\left(\left\{\phi\left(D^{\prime}\right) \mid D^{\prime} \in R(D)\right\}\right) .
$$

Hence $\Phi(D)$ for $D \in \mathcal{D}_{s}$ is the convex hull of the focal points $\phi\left(D^{\prime}\right)$ of all the regulatory domains $D^{\prime}$ having $D$ in their boundary, as defined above, intersected with the threshold hyperplane $\operatorname{supp}(D)$ containing the switching domain $D$ (Figure $3)$.

We have shown that

$$
H(x)=\gamma(\Phi(D)-x)
$$

which is a compact way of writing that $H(x)=\left\{y \in \mathbb{R}^{n} \mid \exists \phi \in \Phi(D)\right.$ such that $y=$ $\gamma(\phi-x)\}$. The Filippov vector field is defined by means of the focal set.

If $\Phi(D)=\{\}$, with $D$ a switching domain, solutions will simply cross $D$; otherwise, sliding mode is possible and convergence takes place "in the direction" of $\Phi(D)$. If $\Phi(D) \cap D=\{\}$, solutions eventually leave $D$. In the case where $\Phi(D) \cap D$ is not empty, it can be assimilated to an equilibrium set within $D$ towards which all solutions will converge in the following sense 


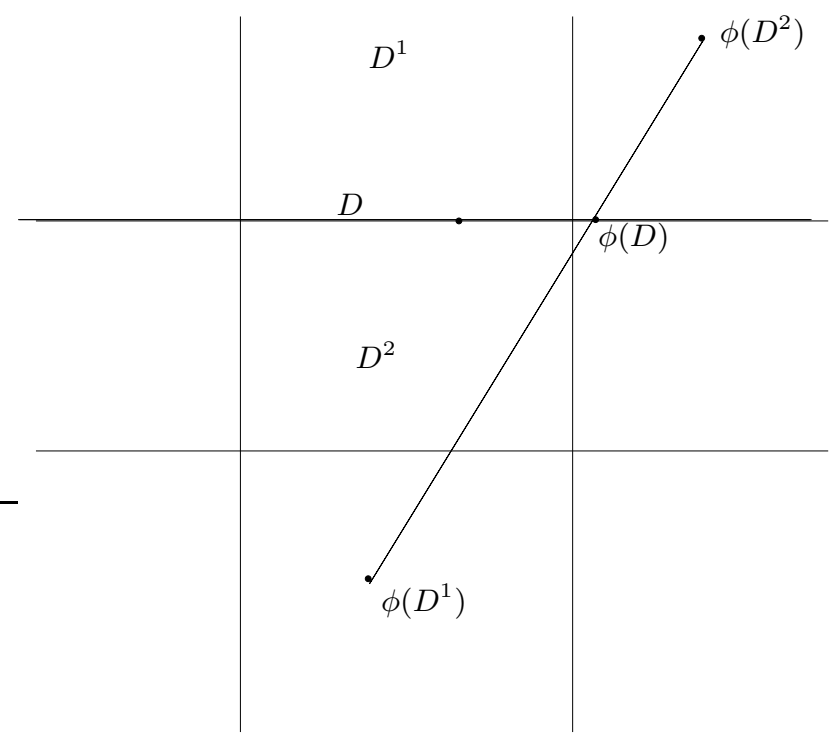

Fig. 3. Illustration of the definition of the focal set on a switching surface $D$ according to the Filippov definition of solutions. The convex hull of the points $\phi\left(D^{1}\right)$ and $\phi\left(D^{2}\right)$ is simply the segment that links them, so that (7) implies that $\phi(D)$ is the intersection of this segment with $\operatorname{supp}(D)$.

Lemma 1. [3] For every regulatory domain $D \in \mathcal{D}_{r}$, all solutions $\xi_{t}$ in D monotonically converge towards the focal set $\Phi(D)$. For every switching domain $D \in \mathcal{D}_{s}$, the non-switching component $\left(\xi_{t}\right)_{i}$ of the solution $\xi_{t}$ in D monotonically converges towards the closed interval

$$
\pi_{i}(\Phi(D))=\left\{\phi_{i} \in \Omega_{i} \mid \phi \in \Phi(D)\right\},
$$

the projection of $\Phi(D)$ onto $\Omega_{i}$, if $\left(\xi_{0}\right)_{i} \notin \pi_{i}(\Phi(D))$. Every switching component $\left(\xi_{t}\right)_{i}$ of the solution $\xi_{t}$ in $D$ is a constant $\left(\xi_{t}\right)_{i}=\pi_{i}(\Phi(D))=\theta_{i}^{q_{i}}$.

Basically, this means that convergence does not take place towards $\Phi(D)$, but towards the smallest hyper-rectangle that contains $\Phi(D)$. Indeed, if $\Phi(D)$ is neither empty, nor a singleton, and $\xi_{t_{0}}$ belongs to $\Phi(D)$, the Filippov vector field at this point is defined as $H\left(\xi_{t_{0}}\right)=\gamma\left(\Phi(D)-\xi_{t_{0}}\right)$ and there is no guarantee that no element of $H\left(\xi_{t_{0}}\right)$ points outside of $\Phi(D)$ (we know however that a solution stays at $\xi_{t_{0}}$ ). Due to the structure of the differential equations, it is on the other hand certain that the transient solution does not leave the smallest hyper-rectangle containing $\Phi(D)$. This phenomenon is illustrated in Figure 4

We then have the following corollary

Corollary 1. [3] All solutions $\xi_{t}$ in D converge towards $\Pi(D)$, if $\xi_{0} \notin \Pi(D)$. For all solutions $\xi_{t}$ in $D, \Pi(D)$ is invariant. 


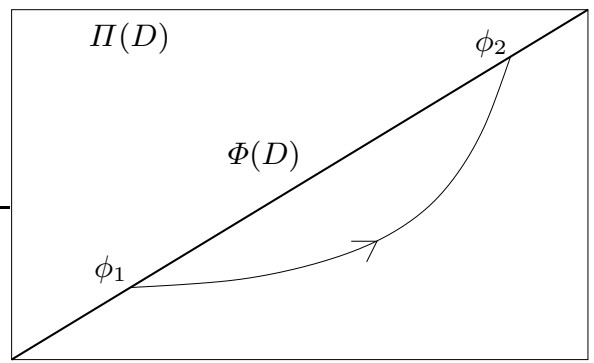

Fig. 4. Illustration of the non invariance of $\Phi(D)$ : solutions with initial condition on $\Phi(D)$ stay inside the box $\Pi(D)$ but do not necessarily stay in $\Phi(D)$

Adding the following assumption

Assumption 1 For all domains $D \in \mathcal{D}$,

$$
\Phi(D) \cap \operatorname{supp}\left(D^{\prime}\right)=\{\}, \forall D^{\prime} \subseteq \partial D .
$$

it has been possible to develop stability results for this family of systems.

\section{Stability and qualitative properties of PL models}

The stability analysis of the various equilibria is a direct consequence of the analysis in the previous section. It is easily seen that equilibria $\bar{x}_{r}$ in some $D \in \mathcal{D}_{r}$ are asymptotically stable. Indeed, they are the focal points of the domains in which they are contained, so that the convergence that was described in the previous section, leads to asymptotic stability. The more difficult part consists in defining and handling the stability of Filippov equilibria that lie in switching surfaces.

In a switching domain $D \in \mathcal{D}_{s}$, recall that solutions are defined by considering the differential inclusion $H(x)$. We say that a point $y \in \Omega$ is an equilibrium point for the differential inclusion if

$$
0 \in H(y),
$$

where $H$ is computed using the Filippov construction in (6). In other words, there is a solution in the sense of Filippov, $\xi_{t}$, such that $\xi_{t}(y)=y, \forall t>0$. We call such a point a singular equilibrium point. It is easily seen that, for $y$ to be an equilibrium point inside $D$, it must belong to $\Phi(D)$. Also, since Assumption 1 prevents $\Phi(D)$ from intersecting the border of $D$, we then have that $\Phi(D) \subset D$. Every element $\phi$ of $\Phi(D)$ is then an equilibrium when $\Phi(D) \subset D$ so that, for every $\phi \in \Phi(D)$, there exists a solution $\xi_{t}(\phi)=\phi$ for all $t$.

One of the interesting results of [3] concerns the link between the configuration of the state transition graph and the stability of an equilibrium. This discrete, qualitative description of the dynamics of the PL system that underlies the qualitative simulation of genetic regulatory networks was originally due to Glass. It indicates the passages 
between the different domains making up the phase space. A state transition graph is a directed graph whose vertices are the domains of the system and whose edges are the possible transitions between these domains (easily determined by examining the PL model [3]). The transition graph of system (1) is illustrated in Figure 5.

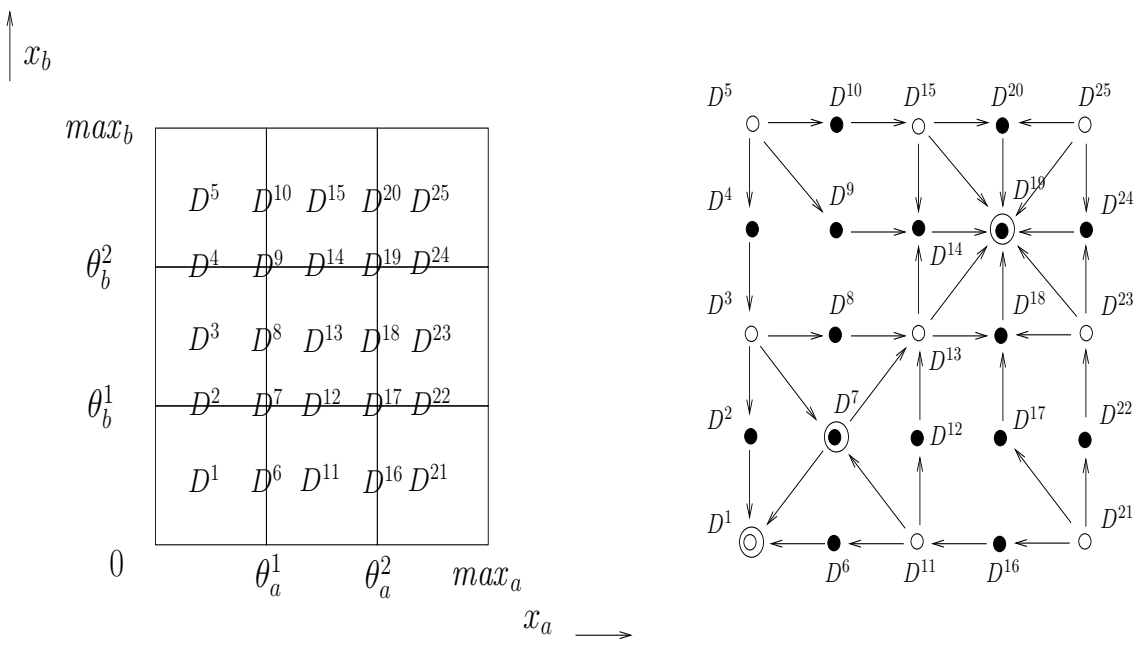

Fig. 5. Subdivision of the state-space in 25 domains and transition graph of system (1)

For a two-dimensional system, we show how this graph indicates the stability of singular equilibria:

Theorem 1. [3] Let the dimension of the PL model be 2, and let D be a switching domain containing a singular equilibrium point $\phi(D)$. If for all regulatory domains $D^{\prime} \in R(D)$ (that is, adjacent to $D$ ), there exists a transition from $D^{\prime}$ to $D$ in the state transition graph, then $\phi(D)$ is asymptotically stable.

This result is purely qualitative: the actual value of the parameters is not needed. It can be directly applied to show that the singular equilibrium $\left(x_{a}, x_{b}\right)=\left(\theta_{a}^{2}, \theta_{b}^{2}\right)$, corresponding to $D^{19}$ on Figure 5, is asymptotically stable because there are transitions to $D^{19}$ from $D^{13}, D^{15}, D^{23}$ and $D^{25}$, the regulatory domains adjacent to $D^{19}$.

A generalization, but in a weaker form, of this theorem to dimension $n$ is also available.

Theorem 2. Assume $\Omega \subset \mathbb{R}^{n}$. Let $D \in \mathcal{D}_{s}$ be a switching domain of order $p \geq 1$ containing a singular equilibrium set $\Phi(D)$ that satisfies Assumption 1. If for all $D^{\prime} \in R(D)$, there is a transition from $D^{\prime}$ to $D$ in the state transition graph, then $\Pi(D)$ is asymptotically stable. 
These results are very helpful for the qualitative analysis of the genetic regulatory networks. However, some stable equilibria cannot be identified through those criteria. Some less restrictive criteria are therefore under development.

Besides this method, we can discover stable equilibria that would not have been directly identified by our criteria, through a rigorous simplification of the model. This can be done through model reduction or identification of regions of the statespace that cannot be reached by the solutions (maybe after some finite time). We will illustrate the kind of things that can be done on an example in the following section. In that section, since the resulting models are very simple, we do not need to go back to transition graph analysis at the end of the reduction procedure, but we could have done so, and it will be necessary to do so if the model reduction procedure does not yield very small models.

\section{Carbon starvation response of $E$. coli}

We will present a specific model reduction and stability analysis for the model in dimension 6 of the carbon starvation response of E. coli of Ropers et al. [29]. In their natural environment, bacteria like Escherichia coli rarely encounter conditions allowing continuous, balanced growth. While nutrients are available, E. coli cells grow quickly, leading to an exponential increase of their biomass, a state called exponential phase. However, upon depletion of an essential nutrient, the bacteria are no longer able to maintain fast growth rates, and the population consequently enters a non-growth state, called stationary phase (Figure 6). During the transition from exponential to stationary phase, each individual $E$. coli bacterium undergoes numerous physiological changes, concerning among other things the morphology and the metabolism of the cell, as well as gene expression [19]. These changes enable the cell to survive prolonged periods of starvation and be resistant to multiple stresses. This carbon starvation response can be reversed and growth resumed, as soon as carbon sources become available again.

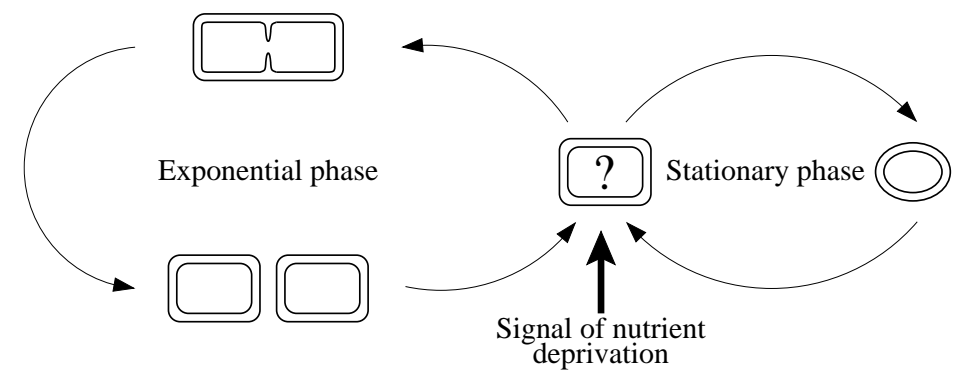

Fig. 6. Nutrient-stress response of bacteria during the transition from exponential to stationary phase. 
On the molecular level, the transition from exponential phase to stationary phase is controlled by a complex genetic regulatory network integrating various environmental signals $[18,24,32]$. The molecular basis of the adaptation of the growth of $E$. coli to carbon starvation conditions has been the focus of extensive studies for decades [18]. However, notwithstanding the enormous amount of information accumulated on the genes, proteins, and other molecules known to be involved in the stress adaptation process, there is currently no global understanding of how the response of the cell emerges from the network of molecular interactions. Moreover, with some exceptions $[1,16,31]$, numerical values for the parameters characterizing the interactions and the molecular concentrations are absent, which makes it difficult to apply traditional methods for the dynamical modeling of genetic regulatory networks.

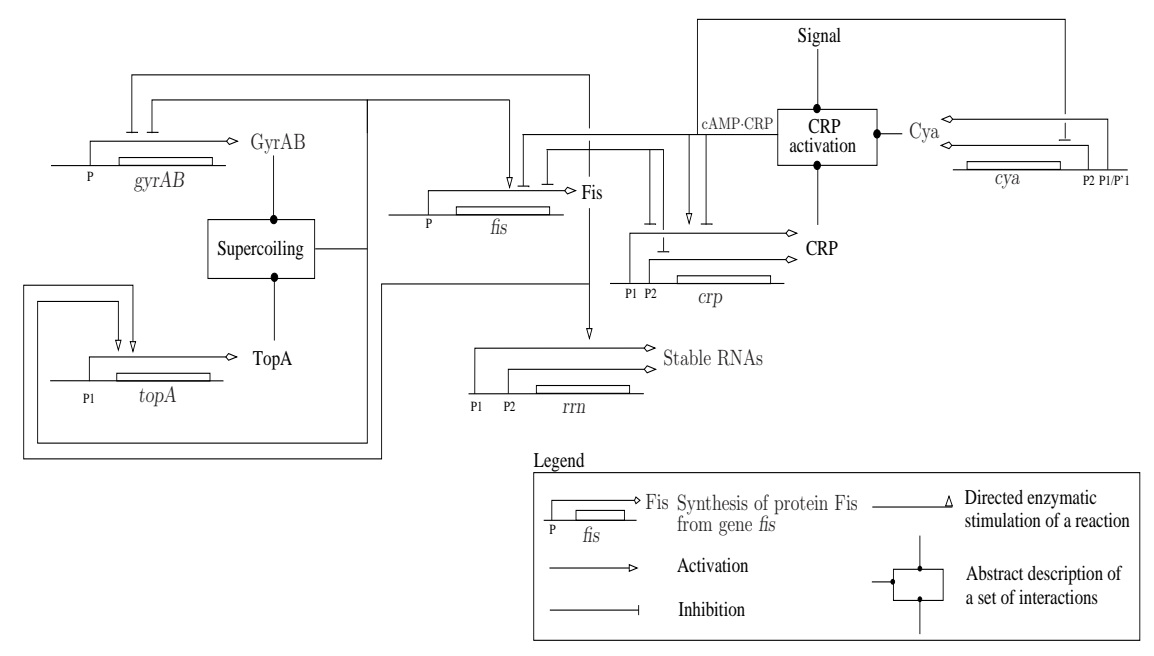

Fig. 7. Network of key genes, proteins, and regulatory interactions involved in the carbon starvation network in E. coli. The notation follows, in a somewhat simplified form, the graphical conventions proposed by Kohn [23]. The contents of the boxes labeled 'Activation' and 'Supercoiling' are detailed in [29].

The above circumstances have motivated the qualitative analysis of the carbon starvation response network in E. coli [29]. The objective of the study was to simulate the response of an $E$. coli bacterium to the absence or presence of carbon sources in the growth medium. To this end, an initial, simple model of the carbon starvation response network has been built on the basis of literature data. It includes six genes that are believed to play a key role in the carbon starvation response (Figure 7). More specifically, the network includes genes encoding proteins whose activity depends on the transduction of the carbon starvation signal (the global regulator $\mathrm{crp}$ and the 
adenylate cyclase $c y a$ ), genes involved in the metabolism (the global regulator fis), cellular growth (the $r r n$ genes coding for stable RNAs), and DNA supercoiling, an important modulator of gene expression (the topoisomerase topA and the gyrase $g y$ $r A B)$.

\subsection{Model of carbon starvation response}

The graphical representation of the network has been translated into a PL model supplemented with parameter inequality constraints. The resulting model consists of seven variables, one concentration variable for the product of each of the six genes $\left(\left(x_{c}, x_{y}, x_{f}, x_{g}, x_{t}, x_{r}\right)\right.$ for (crp, cya, fis, gyrAB, topA, rrn)) and one input variable $u_{s}$ representing the presence or absence of a carbon starvation signal [29]. The 38 parameters are constrained by 54 parameter inequalities, the choice of which is largely determined by experimental data.

The model of Ropers et al. is:

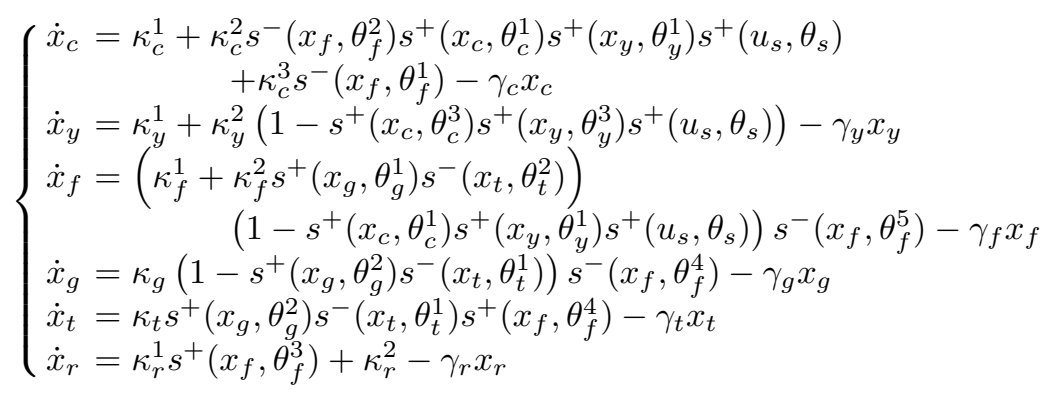

with $u_{s}=0$ in the presence of carbon sources and $u_{s}=1$ in a depleted environment (and $\theta_{s}=0.5$ ). In order to uniquely determine the situation of the various focal points in the state-space, the following constraints on the parameters are needed:

$$
\left\{\begin{array}{lc}
0<\theta_{c}^{1}<\theta_{c}^{2}<\theta_{c}^{3}<\max _{c}, \theta_{c}^{1}<\frac{\kappa_{c}^{1}}{\gamma_{c}}<\theta_{c}^{2}, \theta_{c}^{1}<\frac{\left(\kappa_{c}^{1}+\kappa_{c}^{2}\right)}{\gamma_{c}}<\theta_{c}^{2}, & \theta_{c}^{3}<\frac{\left(\kappa_{c}^{1}+\kappa_{c}^{3}\right)}{\gamma_{c}}<\max _{c} \\
0<\theta_{y}^{1}<\theta_{y}^{2}<\theta_{y}^{3}<\max _{y}, \theta_{y}^{1}<\frac{\kappa_{y}^{1}}{\gamma_{y}}<\theta_{y}^{2}, \theta_{y}^{3}<\frac{\left(\kappa_{y}^{1}+\kappa_{y}^{2}\right)}{\gamma_{y}}<\max _{y} \\
0<\theta_{f}^{1}<\theta_{f}^{2}<\theta_{f}^{3}<\theta_{f}^{4}<\theta_{f}^{5}<\max _{f}, \theta_{f}^{1}<\frac{\kappa_{f}^{1}}{\gamma_{f}}<\theta_{f}^{2} \\
\theta_{f}^{5}<\frac{\left(\kappa_{f}^{1}+\kappa_{f}^{2}\right)}{\gamma_{f}}<\max _{f} \\
0<\theta_{g}^{1}<\theta_{g}^{2}<\max _{g}, \theta_{g}^{2}<\frac{\kappa_{g}}{\gamma_{g}}<\max _{g} \\
0<\theta_{t}^{1}<\theta_{t}^{2}<\max _{t}, \theta_{t}^{2}<\frac{\kappa_{t}}{\gamma_{t}}<\max _{t} \\
0<\theta_{r}<\max _{r}, 0<\frac{\kappa_{r}^{2}}{\gamma_{r}}<\theta_{r}, \theta_{r}<\frac{\left(\kappa_{r}^{1}+\kappa_{r}^{2}\right)}{\gamma_{r}}<\max _{r}
\end{array}\right.
$$

A qualitative analysis of this model has been carried out in [29] by using GNA ( $G e$ netic Network Analyzer [6]), a computer tool that automatically generates the statetransition graph and possible trajectories in that graph, that is, qualitative solutions that are possible for this system. The following simulations are produced for the transition to the stationary phase (Figure 8) and to the exponential phase (Figure 9). In 
the first case, we see that the solution converges towards a single region of the state space, where we can guess that convergence towards an equilibrium takes place. In the second case, the behavior of the solution is not as clear: oscillations can be detected between various regions but it is impossible to say, based on the transition graph alone, if those oscillations are damped or not. Therefore, it is useful to try and analyze the model further to check what kind of oscillations take place (and in the same time if convergence actually takes place towards an equilibrium in the case of the entry in stationary phase).

\subsection{Asymptotic dynamics}

Since the 6-dimensional model, with all its constraints, is too complex to handle directly, we first check if some kind of simplifications can be made. Independently of the case that we will study (stationary phase or exponential phase conditions), we notice that

- $x_{r}$ is a variable whose evolution depends on, but does not influence the rest of the system. As a consequence, it can be removed from the analysis. Once the analysis of the remaining 5-dimensional system is completed, we will be able to easily identify the consequence of its behavior on the concentration of stable RNAs $\left(x_{r}\right)$.

- There exists a finite time after which $x_{t}(t) \leq \theta_{t}^{1}$ since, as long as $x_{t}>\theta_{t}^{1}$, the $x_{t}$ dynamics reduces to

$$
\dot{x}_{t}=-\gamma_{t} x_{t}
$$

Once $x_{t}$ reaches $\theta_{t}^{1}$, we cannot a priori rule out a sliding mode along $x_{t}=\theta_{t}^{1}$. Since $\theta_{t}^{1}<\theta_{t}^{2}$, this indicates that we can replace $s^{-}\left(x_{t}, \theta_{t}^{2}\right)$ with 1 for the purpose of our analysis. We simply consider that the aforementioned finite time has already occurred.

- Similar studies show that $x_{c}(t) \geq \theta_{c}^{1}$ and $x_{y}(t) \geq \theta_{y}^{1}$ after some finite time. We can then replace $s^{+}\left(x_{c}, \theta_{c}^{1}\right)$ and $s^{+}\left(x_{y}, \theta_{y}^{1}\right)$ with 1 in our analysis.

The system that we need to analyze has now become

$$
\left\{\begin{array}{l}
\dot{x}_{c}=\kappa_{c}^{1}+\kappa_{c}^{2} s^{-}\left(x_{f}, \theta_{f}^{2}\right) s^{+}\left(u_{s}, \theta_{s}\right)+\kappa_{c}^{3} s^{-}\left(x_{f}, \theta_{f}^{1}\right)-\gamma_{c} x_{c} \\
\dot{x}_{y}=\kappa_{y}^{1}+\kappa_{y}^{2}\left(1-s^{+}\left(x_{c}, \theta_{c}^{3}\right) s^{+}\left(x_{y}, \theta_{y}^{3}\right) s^{+}\left(u_{s}, \theta_{s}\right)\right)-\gamma_{y} x_{y} \\
\dot{x}_{f}=\left(\kappa_{f}^{1}+\kappa_{f}^{2} s^{+}\left(x_{g}, \theta_{g}^{1}\right)\right) s^{-}\left(u_{s}, \theta_{s}\right) s^{-}\left(x_{f}, \theta_{f}^{5}\right)-\gamma_{f} x_{f} \\
\dot{x}_{g}=\kappa_{g}\left(1-s^{+}\left(x_{g}, \theta_{g}^{2}\right) s^{-}\left(x_{t}, \theta_{t}^{1}\right)\right) s^{-}\left(x_{f}, \theta_{f}^{4}\right)-\gamma_{g} x_{g} \\
\dot{x}_{t}=\kappa_{t} s^{+}\left(x_{g}, \theta_{g}^{2}\right) s^{-}\left(x_{t}, \theta_{t}^{1}\right) s^{+}\left(x_{f}, \theta_{f}^{4}\right)-\gamma_{t} x_{t}
\end{array}\right.
$$

The next simplification step consists in seeing that $x_{y}$ does not influence the rest of the model, so that it can be removed, and that $x_{c}$ does not influence the rest of the model either (except $x_{y}$ ) so that it can also be removed. These actions are in the same line of thought as the removal of $x_{r}$. As a consequence of these simplifications, we are able to see that the core of the long term dynamics is not really influenced by $x_{r}$, $x_{y}$ and $x_{c}$. We now have the three-dimensional system: 


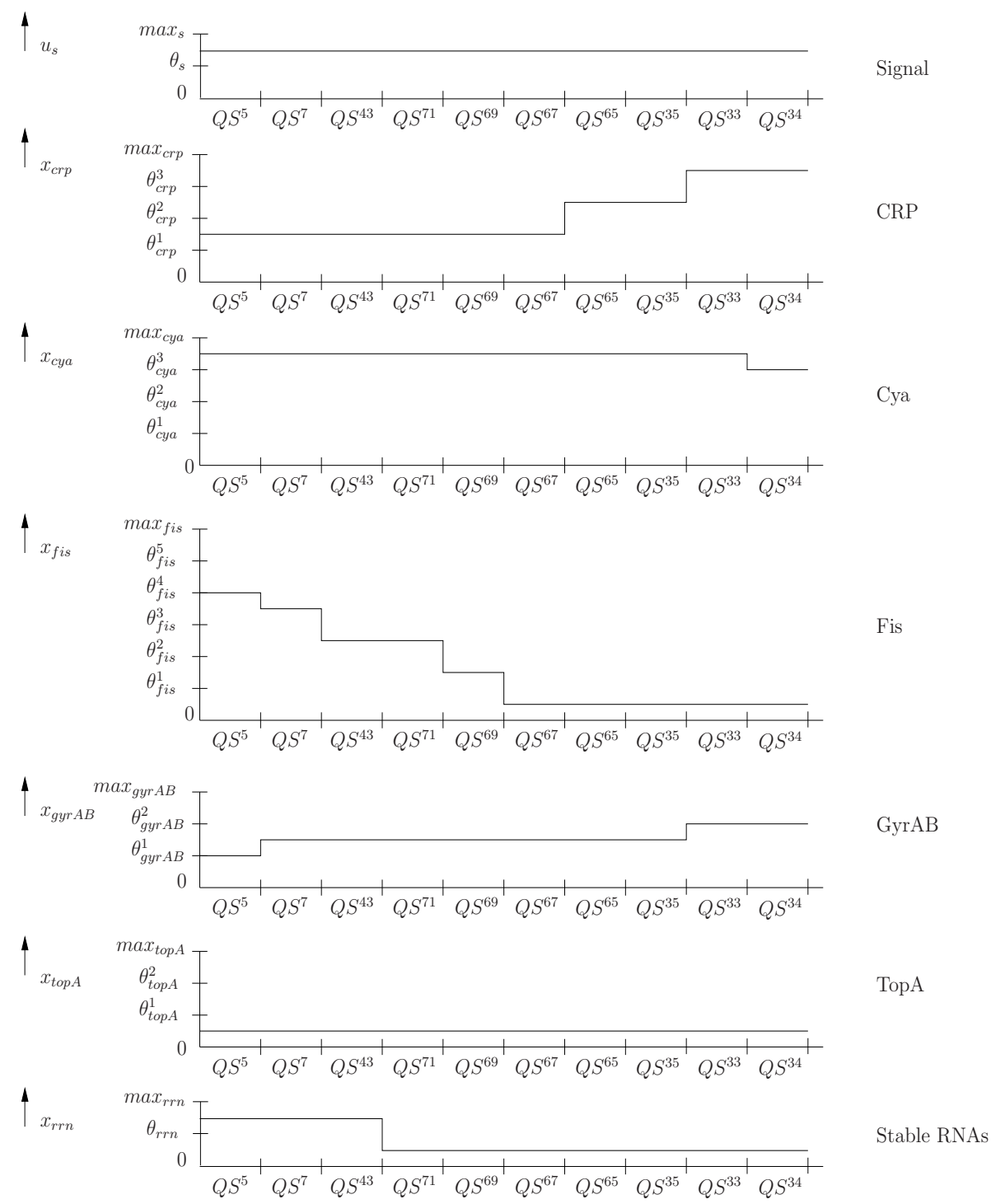

Fig. 8. Entry into stationary phase: qualitative temporal evolution of the proteins and stable RNA concentration in a depleted environment with the organisms being at the equilibrium of the exponential phase at the initial time. Convergence to one domain is detected (the domain where $x_{c}>\theta_{c}^{3}, x_{y}=\theta_{y}^{3}, x_{f}<\theta_{f}^{1}, x_{g}=\theta_{g}^{2}, x_{t}<\theta_{t}^{1}$ and $x_{r}<\theta_{r}$ ) 


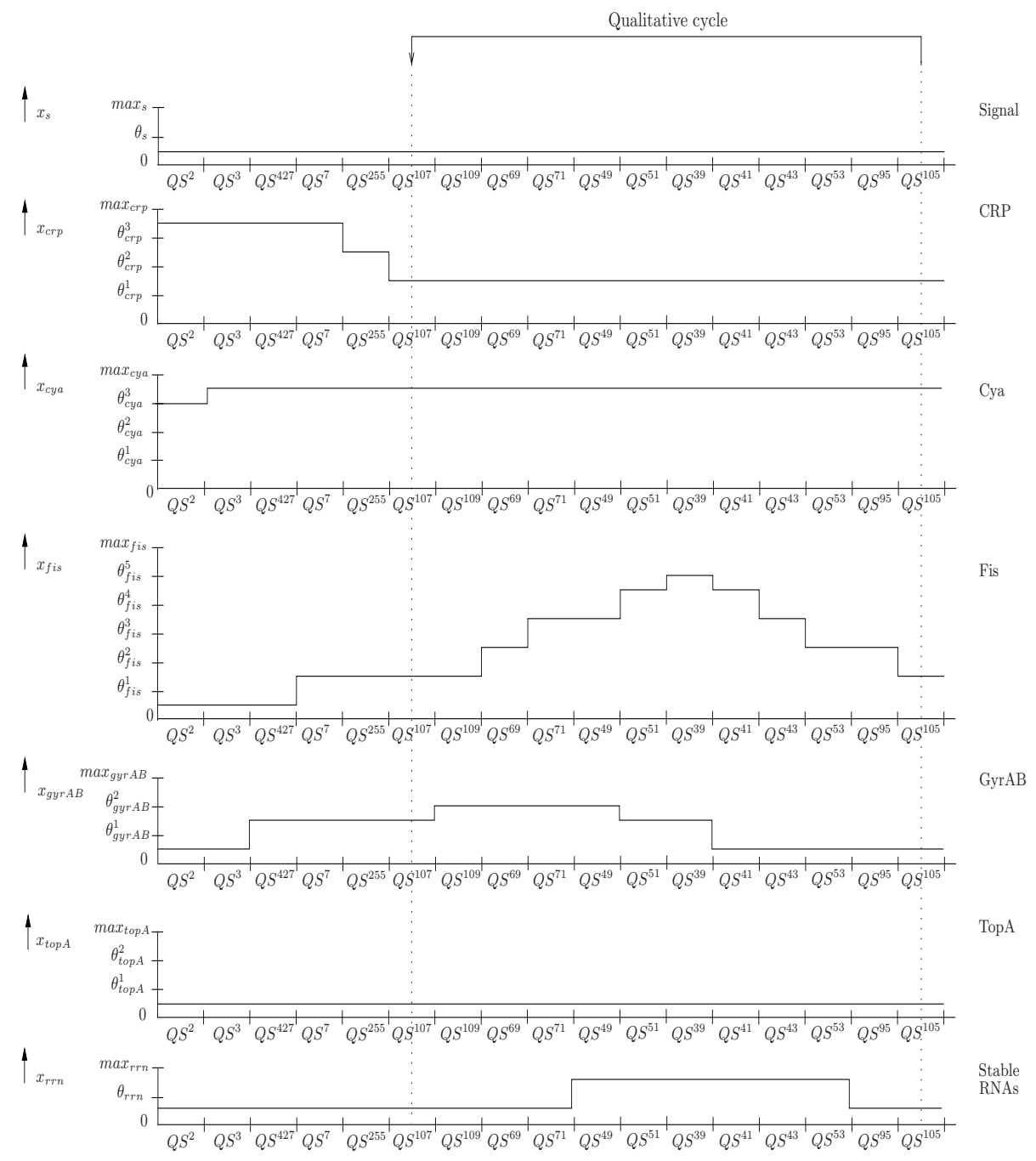

Fig. 9. Entry into exponential phase: qualitative temporal evolution of the proteins and stable RNA concentration in a rich environment with the organisms being at the equilibrium of the stationary phase at the initial time. Oscillations of the $x_{f}$ and $x_{g}$ states is detected. 


$$
\left\{\begin{array}{l}
\dot{x}_{f}=\left(\kappa_{f}^{1}+\kappa_{f}^{2} s^{+}\left(x_{g}, \theta_{g}^{1}\right)\right) s^{-}\left(u_{s}, \theta_{s}\right) s^{-}\left(x_{f}, \theta_{f}^{5}\right)-\gamma_{f} x_{f} \\
\dot{x}_{g}=\kappa_{g}\left(1-s^{+}\left(x_{g}, \theta_{g}^{2}\right) s^{-}\left(x_{t}, \theta_{t}^{1}\right)\right) s^{-}\left(x_{f}, \theta_{f}^{4}\right)-\gamma_{g} x_{g} \\
\dot{x}_{t}=\kappa_{t} s^{+}\left(x_{g}, \theta_{g}^{2}\right) s^{-}\left(x_{t}, \theta_{t}^{1}\right) s^{+}\left(x_{f}, \theta_{f}^{4}\right)-\gamma_{t} x_{t}
\end{array}\right.
$$

Once we have analyzed the behavior of the solutions of this model, we will be able to reconstruct what happens with $x_{c}, x_{y}$ and $x_{r}$. For this analysis, we still suppose that $x_{t} \leq \theta_{t}^{1}$.

\subsection{Asymptotic dynamics in the absence of carbon sources}

The analysis of the case $u_{s}=1$, the stationary phase solution in a depleted environment, is very straightforward. System (11) becomes

$$
\left\{\begin{array}{l}
\dot{x}_{f}=-\gamma_{f} x_{f} \\
\dot{x}_{g}=\kappa_{g}\left(1-s^{+}\left(x_{g}, \theta_{g}^{2}\right) s^{-}\left(x_{t}, \theta_{t}^{1}\right)\right) s^{-}\left(x_{f}, \theta_{f}^{4}\right)-\gamma_{g} x_{g} \\
\dot{x}_{t}=\kappa_{t} s^{+}\left(x_{g}, \theta_{g}^{2}\right) s^{-}\left(x_{t}, \theta_{t}^{1}\right) s^{+}\left(x_{f}, \theta_{f}^{4}\right)-\gamma_{t} x_{t}
\end{array}\right.
$$

so that $x_{f}$ goes to 0 . It is then directly seen that, after a finite time (the time taken for $x_{f}$ to fall below $\theta_{f}^{4}$, we have

$$
\dot{x}_{t}=-\gamma_{t} x_{t}
$$

so that $x_{t}$ also goes to zero. The $x_{g}$ dynamics then reduce to

$$
\dot{x}_{g}=\kappa_{g} s^{-}\left(x_{g}, \theta_{g}^{2}\right)-\gamma_{g} x_{g}
$$

so that $x_{g}$ reaches $\theta_{g}^{2}$ in finite time. The three dimensional system thus has a very simple behavior: the state goes to $\left(x_{f}, x_{g}, x_{t}\right)=\left(0, \theta_{g}^{2}, 0\right)$.

Since the solutions of the 6-dimensional system are bounded, the behavior of the other three states can be deduced from the analysis of the corresponding equations with $\left(x_{f}, x_{g}, x_{t}\right)$ approaching their equilibrium (so that $x_{f}<\theta_{f}^{1}, x_{t}<\theta_{t}^{1}$ and $x_{g}>$ $\theta_{g}^{1}$ ). We then have:

$$
\left\{\begin{array}{l}
\dot{x}_{c}=\kappa_{c}^{1}+\kappa_{c}^{2}+\kappa_{c}^{3}-\gamma_{c} x_{c} \\
\dot{x}_{y}=\kappa_{y}^{1}+\kappa_{y}^{2}\left(1-s^{+}\left(x_{c}, \theta_{c}^{3}\right) s^{+}\left(x_{y}, \theta_{y}^{3}\right)\right)-\gamma_{y} x_{y} \\
\dot{x}_{r}=\kappa_{r}^{2}-\gamma_{r} x_{r}
\end{array}\right.
$$

It is then directly seen that, once $\left(x_{f}, x_{g}, x_{t}\right)$ is close to its equilibrium, the variables $\left(x_{c}, x_{r}\right)$ exponentially converge towards $\left(\frac{\kappa_{c}^{1}+\kappa_{c}^{2}+\kappa_{c}^{3}}{\gamma_{c}}, \frac{\kappa_{r}^{2}}{\gamma_{r}}\right)$ while $x_{y}$ reaches $\theta_{y}^{3}$ in finite time.

\subsection{Asymptotic dynamics in the presence of carbon sources}

The case $u_{s}=0$, the behavior of the model in an environment rich in carbon sources, is more intricate to analyze. System (11) becomes 


$$
\left\{\begin{array}{l}
\dot{x}_{f}=\left(\kappa_{f}^{1}+\kappa_{f}^{2} s^{+}\left(x_{g}, \theta_{g}^{1}\right)\right) s^{-}\left(x_{f}, \theta_{f}^{5}\right)-\gamma_{f} x_{f} \\
\dot{x}_{g}=\kappa_{g}\left(1-s^{+}\left(x_{g}, \theta_{g}^{2}\right) s^{-}\left(x_{t}, \theta_{t}^{1}\right)\right) s^{-}\left(x_{f}, \theta_{f}^{4}\right)-\gamma_{g} x_{g} \\
\dot{x}_{t}=\kappa_{t} s^{+}\left(x_{g}, \theta_{g}^{2}\right) s^{-}\left(x_{t}, \theta_{t}^{1}\right) s^{+}\left(x_{f}, \theta_{f}^{4}\right)-\gamma_{t} x_{t}
\end{array}\right.
$$

As stated earlier, we know that $x_{t} \leq \theta_{t}^{1}$ after some finite time; this does not help us for further simplifications of this model. In the following, we will show that, after some finite-time, we have $x_{t}<\theta_{t}^{1}$, which will help us eliminate the $x_{t}$ equation. In order to do that, we first show that, after some finite time, $x_{g} \leq \theta_{g}^{2}$.

Indeed, if we suppose that $x_{g}>\theta_{g}^{2}$ for all times, system (11) would become

$$
\left\{\begin{array}{l}
\dot{x}_{f}=\left(\kappa_{f}^{1}+\kappa_{f}^{2}\right) s^{-}\left(x_{f}, \theta_{f}^{5}\right)-\gamma_{f} x_{f} \\
\dot{x}_{g}=\kappa_{g} s^{+}\left(x_{t}, \theta_{t}^{1}\right) s^{-}\left(x_{f}, \theta_{f}^{4}\right)-\gamma_{g} x_{g} \\
\dot{x}_{t}=\kappa_{t} s^{-}\left(x_{t}, \theta_{t}^{1}\right) s^{+}\left(x_{f}, \theta_{f}^{4}\right)-\gamma_{t} x_{t}
\end{array}\right.
$$

which shows that $x_{f}$ reaches $\theta_{f}^{5}$ in finite time so that $\dot{x}_{g}$ becomes equal to

$$
\dot{x}_{g}=-\gamma_{g} x_{g}
$$

This leads to the convergence of $x_{g}$ to 0 and thus to below $\theta_{g}^{2}$, which is a contradiction. This shows that $x_{g}$ should reach $\theta_{g}^{2}$ in finite time when $x_{g}(0)>\theta_{g}^{2}$.

An ensuing case-by-case analysis shows that the region where $x_{g} \leq \theta_{g}^{2}$ is invariant [2].

We will now show that $x_{t}$ is decreasing almost all of the time when $x_{g} \leq \theta_{g}^{2}$ and $x_{t} \leq \theta_{t}^{1}$, that is in a region which we have shown to be reached in finite time and invariant. Detailing three cases, we have:

$x_{g}<\theta_{g}^{2}$ or $x_{f}<\theta_{f}^{4}: \dot{x}_{t}=-\gamma_{t} x_{t}$.

$x_{g}=\theta_{g}^{2}$ and $x_{f}>\theta_{f}^{4}$ : We have $\dot{x}_{g}=-\gamma_{g} x_{g}<0$ at such a point and in a neighbor-

hood surrounding each such point so that any solution directly enters the region where $x_{g}<\theta_{g}^{2}$ (and consequently $\dot{x}_{t}=-\gamma_{t} x_{t}$, as we have seen).

$x_{g}=\theta_{g}^{2}$ and $x_{f}=\theta_{f}^{4}$ : We have $\dot{x}_{f}=\left(\kappa_{f}^{1}+\kappa_{f}^{2}\right)-\gamma_{f} x_{f}>0$ at this point and in a neighborhood surrounding it, so that any solution directly goes in one of the two previously described regions, where we have seen that $x_{t}$ is decreasing.

For any solution of (11), $x_{t}$ could only increase if $x$ stayed in the second or third region, which we have shown not to be possible. We then have $\dot{x}_{t}=-\gamma_{t} x_{t}$ for almost all times in the region of interest. After elimination of $x_{t}$, we have to analyze the following system:

$$
\left\{\begin{array}{l}
\dot{x}_{f}=\left(\kappa_{f}^{1}+\kappa_{f}^{2} s^{+}\left(x_{g}, \theta_{g}^{1}\right)\right) s^{-}\left(x_{f}, \theta_{f}^{5}\right)-\gamma_{f} x_{f} \\
\dot{x}_{g}=\kappa_{g} s^{-}\left(x_{g}, \theta_{g}^{2}\right) s^{-}\left(x_{f}, \theta_{f}^{4}\right)-\gamma_{g} x_{g}
\end{array}\right.
$$

At first sight, this analysis is not straightforward because this is a second order piecewise linear system with two thresholds in each direction, which theoretically gives rise to 9 regions. However, as is illustrated on Figure 10, some of the regions have the same dynamics and can be grouped together, giving rise to six regions. 


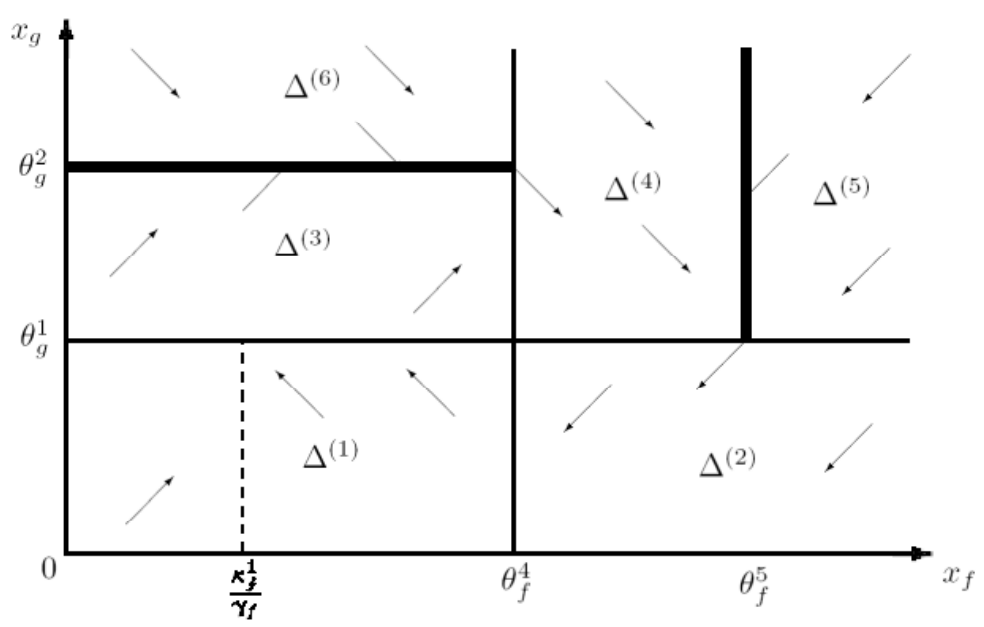

Fig. 10. Illustration of the vector field and the various regions for system (12). The thick black lines indicate where sliding modes can occur.

The behavior of the solutions along the thick black lines, where sliding modes are present, can be directly inferred from the Filippov construction. However, simple observations indicate what actually happens: along the line where $x_{g}=\theta_{g}^{2}$ and $x_{f}<$ $\theta_{f}^{4}$, we have

$$
\dot{x}_{g}=\kappa_{g} s^{-}\left(x_{g}, \theta_{g}^{2}\right)-\gamma_{g} x_{g}
$$

with

$$
\frac{\kappa_{g}}{\gamma_{g}}>\theta_{g}^{2}
$$

so that the line is attractive (black wall). Moreover,

$$
\dot{x}_{f}=\left(\kappa_{f}^{1}+\kappa_{f}^{2}\right)-\gamma_{f} x_{f}>0
$$

so that $x_{f}$ is increasing and all solutions reach the end-point $\left(x_{f}, x_{g}\right)=\left(\theta_{f}^{4}, \theta_{g}^{2}\right)$ in finite time. In some sense, each time the solution reaches this black wall, there is a reset taking place that sends the system to the end-point $\left(\theta_{f}^{4}, \theta_{g}^{2}\right)$

Along the line where $x_{f}=\theta_{f}^{5}$ and $x_{g}>\theta_{g}^{1}$, we have

$$
\dot{x}_{f}=\left(\kappa_{f}^{1}+\kappa_{f}^{2}\right) s^{-}\left(x_{f}, \theta_{f}^{5}\right)-\gamma_{f} x_{f}
$$

so that this line also is a black wall (bearing in mind that $\frac{\kappa_{f}^{1}+\kappa_{f}^{2}}{\gamma_{f}}>\theta_{f}^{5}$ ). In addition,

$$
\dot{x}_{g}=-\gamma_{g} x_{g}
$$

so that $x_{g}$ is decreasing and all solutions reach the end-point $\left(x_{f}, x_{g}\right)=\left(\theta_{f}^{5}, \theta_{g}^{1}\right)$ in finite time. 
The observation of Figure 10 (as well as a detailed analysis of the linear systems in each of the regions) indicate that, eventually, the solutions oscillate around $\left(x_{f}, x_{g}\right)=\left(\theta_{f}^{4}, \theta_{g}^{1}\right)$. Whether this oscillation is damped, neutrally stable or unstable is still unclear. It is clear, though, that the oscillation is bounded, as it cannot go beyond the black walls.

In order to analyze the oscillations, we will compute the first return map from and to the segment that links $\left(\theta_{f}^{4}, \theta_{g}^{1}\right)$ to $\left(\theta_{f}^{4}, \theta_{g}^{2}\right)$. We will therefore consider some $\left(\theta_{f}^{4}, x\right)$ as initial condition and compute the function $f(x)$ such that $\left(\theta_{f}^{4}, f(x)\right)$ is the image of $\left(\theta_{f}^{4}, x\right)$ on the segment after one cycle around $\left(\theta_{f}^{4}, \theta_{g}^{1}\right)$. The computation of this first-return map can be handled in four steps, corresponding to the passages in the four regions surrounding $\left(\theta_{f}^{4}, \theta_{g}^{1}\right)$.

The first step consists in computing the image of $\left(\theta_{f}^{4}, x\right)$, belonging to the initial segment, on the horizontal segment that links $\left(\theta_{f}^{4}, \theta_{g}^{1}\right)$ to $\left(\theta_{f}^{5}, \theta_{g}^{1}\right)$. The transition takes place in the region $\Delta^{(4)}$ so that (12) becomes

$$
\left\{\begin{array}{l}
\dot{x}_{f}=\kappa_{f}^{1}+\kappa_{f}^{2}-\gamma_{f} x_{f} \\
\dot{x}_{g}=-\gamma_{g} x_{g}
\end{array}\right.
$$

whose solution is

$$
\left\{\begin{array}{l}
x_{f}(t)=\theta_{f}^{4} e^{-\gamma_{f} t}+\frac{\kappa_{f}^{1}+\kappa_{f}^{2}}{\gamma_{f}}\left(1-e^{-\gamma_{f} t}\right) \\
x_{g}(t)=x e^{-\gamma_{g} t}
\end{array}\right.
$$

In a first computation, we will suppose the absence of the vertical black wall, and use the dynamics (13) for both regions $\Delta^{(4)}$ and $\Delta^{(5)}$ : it is then straightforward to see that the solution impacts the target segment when $x_{g}(t)=\theta_{g}^{1}$, that is, at

$$
\begin{aligned}
& t=t_{1}(x)=\frac{\ln (x)-\ln \left(\theta_{g}^{1}\right)}{\gamma_{g}}, \text { so that } \\
& \qquad \begin{aligned}
x_{f}\left(t_{1}(x)\right) & =\theta_{f}^{4} e^{-\gamma_{f} t_{1}(x)}+\frac{\kappa_{f}^{1}+\kappa_{f}^{2}}{\gamma_{f}}\left(1-e^{-\gamma_{f} t_{1}(x)}\right) \\
& =\theta_{f}^{4}\left(\frac{\theta_{g}^{1}}{x}\right)^{\frac{\gamma_{f}}{\gamma_{g}}}+\frac{\kappa_{f}^{1}+\kappa_{f}^{2}}{\gamma_{f}}\left(1-\left(\frac{\theta_{g}^{1}}{x}\right)^{\frac{\gamma_{f}}{\gamma_{g}}}\right)
\end{aligned}
\end{aligned}
$$

However, we must account for the black-wall and it is possible that the actual solution hits this wall before reaching the target segment, so that the previously computed $x_{f}\left(t_{1}(x)\right)>\theta_{f}^{5}$. In that case, the actual solution stays on the vertical black wall until it reaches the point $\left(\theta_{f}^{5}, \theta_{g}^{1}\right)$. Therefore the target of the point $\left(\theta_{f}^{4}, x\right)$ on the horizontal segment is

$$
\left(f_{1}(x), \theta_{g}^{1}\right)=\left(\min \left(\theta_{f}^{4}\left(\frac{\theta_{g}^{1}}{x}\right)^{\frac{\gamma_{f}}{\gamma_{g}}}+\frac{\kappa_{f}^{1}+\kappa_{f}^{2}}{\gamma_{f}}\left(1-\left(\frac{\theta_{g}^{1}}{x}\right)^{\frac{\gamma_{f}}{\gamma_{g}}}\right), \theta_{f}^{5}\right), \theta_{g}^{1}\right)
$$

Similarly we can define $\left(\theta_{f}^{4}, f_{2}(x)\right)$ as the image of $\left(x, \theta_{g}^{1}\right)$ (with $\left.x \in\left[\theta_{f}^{4}, \theta_{f}^{5}\right]\right)$ on the vertical segment below the equilibrium, $\left(f_{3}(x), \theta_{g}^{1}\right)$ as the image of $\left(\theta_{f}^{4}, x\right)$ (with 
$\left.x \in\left[0, \theta_{g}^{1}\right]\right)$ on the horizontal segment on the left of the equilibrium and $\left(\theta_{f}^{4}, f_{4}(x)\right)$ as the image of $\left(x, \theta_{g}^{1}\right)$ (with $x \in\left[0, \theta_{f}^{4}\right]$ ) on the initial segment.

This yields

$$
\begin{aligned}
& f_{1}(x)=\min \left(\theta_{f}^{4}\left(\frac{\theta_{g}^{1}}{x}\right)^{\frac{\gamma_{f}}{\gamma_{g}}}+\frac{\kappa_{f}^{1}+\kappa_{f}^{2}}{\gamma_{f}}\left(1-\left(\frac{\theta_{g}^{1}}{x}\right)^{\frac{\gamma_{f}}{\gamma_{g}}}\right), \theta_{f}^{5}\right) \\
& f_{2}(x)=\theta_{g}^{1}\left(\frac{\theta_{f}^{4}-\frac{\kappa_{f}^{1}}{\gamma_{f}}}{x-\frac{\kappa_{f}^{1}}{\gamma_{f}}}\right)^{\frac{\gamma_{g}}{\gamma_{f}}}\left(\frac{\theta_{g}^{1}-\frac{\kappa_{g}}{\gamma_{g}}}{x-\frac{\kappa_{g}}{\gamma_{g}}}\right)^{\frac{\gamma_{f}}{\gamma_{g}}}+\frac{\kappa_{f}^{1}}{\gamma_{f}}\left(1-\left(\frac{\theta_{g}^{1}-\frac{\kappa_{g}}{\gamma_{g}}}{x-\frac{\kappa_{g}}{\gamma_{g}}}\right)^{\frac{\gamma_{f}}{\gamma_{g}}}\right) \\
& f_{3}(x)=\theta_{f}^{4}\left(\theta_{g}^{1}\left(\frac{\theta_{f}^{4}-\frac{\kappa_{f}^{1}+\kappa_{f}^{2}}{\gamma_{f}}}{x-\frac{\kappa_{f}^{1}+\kappa_{f}^{2}}{\gamma_{f}}}\right)^{\frac{\gamma_{g}}{\gamma_{f}}}+\frac{\kappa_{g}}{\gamma_{g}}\left(1-\left(\frac{\theta_{f}^{4}-\frac{\kappa_{f}^{1}+\kappa_{f}^{2}}{\gamma_{f}}}{x-\frac{\kappa_{f}^{1}+\kappa_{f}^{2}}{\gamma_{f}}}\right)^{\frac{\gamma_{g}}{\gamma_{f}}}\right), \theta_{g}^{2}\right)
\end{aligned}
$$

and $f(x)=f_{4}\left(f_{3}\left(f_{2}\left(f_{1}(x)\right)\right)\right)$ which has $x=\theta_{g}^{1}$ as a fixed point. It was then shown in [2] that $f^{\prime}(x)<1$ when $x>\theta_{f}^{4}$, so that the sequence $x_{n+1}=f\left(x_{n}\right)$, which represents the successive impacts on the initial segment converges to $x=\theta_{f}^{4}$. We can then conclude that the cyclic solutions that surround $\left(\theta_{f}^{4}, \theta_{g}^{1}\right)$ are damped. This point is therefore a globally attractive equilibrium of (12) (cf. [9] that gives more general results in $n$ dimensions for a negative feedback loop).

Having elucidated the dynamical behavior of the $\left(x_{f}, x_{g}\right)$ subsystem, we can now deduce the behavior of all other states. From the moment that we have $x_{g}<\theta_{g}^{2}$, it comes from (11) that

$$
\dot{x}_{t}=-\gamma_{t} x_{t}
$$

so that $x_{t}$ goes to 0 . Once those three states are close to their equilibrium value, the remaining three equations become

$$
\left\{\begin{array}{l}
\dot{x}_{c}=\kappa_{c}^{1}-\gamma_{c} x_{c} \\
\dot{x}_{y}=\kappa_{y}^{1}+\kappa_{y}^{2}-\gamma_{y} x_{y} \\
\dot{x}_{r}=\kappa_{r}^{1}+\kappa_{r}^{2}-\gamma_{r} x_{r}
\end{array}\right.
$$

so that convergence of $\left(x_{c}, x_{y}, x_{r}\right)$ towards $\left(\frac{\kappa_{c}^{1}}{\gamma_{c}}, \frac{\kappa_{y}^{1}+\kappa_{y}^{2}}{\gamma_{y}}, \frac{\kappa_{r}^{1}+\kappa_{r}^{2}}{\gamma_{r}}\right)$ takes place.

\subsection{Comparison of the equilibria}

It is interesting to compare both equilibria: we have

\begin{tabular}{|c|c|c|c|c|c|c|}
\hline & $x_{c}$ & $x_{y}$ & $x_{f}$ & $x_{g}$ & $x_{t}$ & $x_{r}$ \\
\hline$u_{s}=1$ & $\frac{\kappa_{c}^{1}+\kappa_{c}^{2}+\kappa_{c}^{3}}{\gamma_{c}}$ & $\theta_{y}^{3}$ & 0 & $\theta_{g}^{2}$ & 0 & $\frac{\kappa_{r}^{2}}{\gamma_{r}}$ \\
\hline$u_{s}=0$ & $\frac{\kappa_{c}^{1}}{\gamma_{c}}$ & $\frac{\kappa_{y}^{1}+\kappa_{y}^{2}}{\gamma_{y}}$ & $\theta_{f}^{4}$ & $\theta_{g}^{1}$ & 0 & $\frac{\kappa_{r}^{1}+\kappa_{r}^{2}}{\gamma_{r}}$ \\
\hline
\end{tabular}


We see that most genes settle at different levels depending on the absence or presence of carbon sources. The most illustrative of the difference between the two states (carbon starved or not) is $x_{r}$, which represents the concentration of stable RNAs and is a good indicator of the cellular growth. As expected, when carbon sources are depleted, the equilibrium level of $x_{r}$ is smaller than when carbon sources are abundant: when carbon shortage occurs, $x_{r}$ stays at a "house-keeping"-level whereas $\frac{\kappa_{r}^{1}+\kappa_{r}^{2}}{\gamma_{r}}$, the equilibrium value in the presence of carbon sources, allows for fast cell growth. Also to be noted is the fact that $x_{t}=0$ in both cases; this does not mean that topA, the gene corresponding to $x_{t}$, is useless. Indeed, when the carbon sources are either continuously present or absent, the effect of topA eventually dies down. However, in a time-varying environment, where nutrients are alternatively present and absent, an increase of the $x_{t}$ concentration can occur whenever $x_{g}>\theta_{g}^{2}$ and $x_{f}>\theta_{f}^{4}$. TopA thus influences the transients.

\subsection{Abstraction of the reduction method}

We have seen that the preliminary model reduction has allowed for a simplification of the model analysis. Indeed, a global stability analysis of a 6-order model is no easy task, whereas there are various methods for the analysis of second order models. The reduction of the dimension of dynamical models is critical in the further development of the mathematical methods for genetic regulatory networks analysis because the networks typically are very large, so that it is rarely possible to study them directly. Classically, it has been attempted to apply time-scale separation methods, but these are mainly efficient for eliminating the fast metabolic components from mixed metabolic-genetic networks. Also balanced truncation methods have been introduced for genetic regulatory networks where inputs signals (action on the network) and output signals (measurements) are clearly identified $([15,21])$. In this example, we have exploited the hierarchical triangular structure of the model arising after a finite time (this finite time allowed us to get rid of some of the interactions interfering with the triangular structure). We notice from graph theory that the identification of such a structure in the graph corresponding to the network is equivalent to the search for the strongly connected components of the graph. There are efficient algorithms to do so on large graphs, so that this model reduction method is tractable for the huge graphs that represent genetic regulatory networks (preliminary work on that subject has been done in [4] with links to GNA). Combining this approach with thresholds elimination allows for a progressive simplification of the graphs.

\section{Challenges in PL models analysis}

One of the major challenges in the analysis of models of genetic regulatory networks lies in the difficulty of obtaining accurate parameters. Therefore, one has to develop methods to identify the qualitative behavior of the system: when the parameters are linked together through inequalities (instead of being fixed at given values), 
we would like to be able to say something about the stability of the equilibria. Some interesting results have been obtained on that subject in [3], as was shown in Section 2 , and we would like to identify other cases where stability results can be deduced.

As we have seen in the analysis of $E$. coli, we are able to mathematically analyze PL models that are not trivial (dimension 6). However, actual genetic regulatory networks are much larger than that. It is therefore of paramount importance to develop methods that will help analyzing such large systems. Two major research directions are explored for that purpose: the model reduction approach (through balancing or through singular perturbations, in the linear case) and the separation of the original model into smaller, interconnected pieces that can be easily analyzed, as we have shown here.

Moreover, experimental techniques (e.g. gene deletion) are now available and allow to modify the production or degradation terms of some genes of the networks. This leads to problems of mathematical control of piecewise affine genetic networks, similar to more general problems for hybrid affine systems [14]. The global problem is to control the trajectories through some prescribed sequence of rectangular regions. Some preliminary results have been obtained in [8]. For example, we have shown that a simple two-gene inhibitor system with a single equilibrium can be controlled to a bistable switch. We believe that interesting and original control problems are still to be solved in this domain.

\section{Acknowledgments}

This research is supported by the HYGEIA project of the NEST-Adventure program of the European Union (NEST-004995) on Hybrid Systems for Biochemical Network Modeling and Analysis.

\section{References}

1. J. Botsford and J. Harman. Cyclic AMP in prokaryotes. Microbiological reviews, 56(1):100-122, 1992.

2. K. Bouraima Madjebi. Etude de modèles de réseau de régulation génique. Master's thesis, University of Orsay, 2005.

3. R. Casey, H. de Jong, and J.-L. Gouzé. Piecewise-linear models of genetic regulatory networks: Equilibria and their stability. J. Math. Biol., 52:27-56, 2006.

4. D. Cristescu. Algorithmic study on genetic regulatory networks. Technical report, Automatic control and computer science faculty, Politechnica University of Bucharest, 2006. internship report.

5. H. de Jong. Modeling and simulation of genetic regulatory systems: a literature review. J. Comput. Biol., 9:67-103, 2002.

6. H. de Jong, J. Geiselmann, C. Hernandez, and M. Page. Genetic Network Analyzer: Qualitative simulation of genetic regulatory networks. Bioinformatics, 19(3):336-344, 2003. 
7. H. de Jong, J.-L. Gouzé, C. Hernandez, M. Page, T. Sari, and J. Geiselmann. Qualitative simulation of genetic regulatory networks using piecewise-linear models. Bull. Math. Biol., 6:301-340, 2004.

8. E. Farcot and J.-L. Gouzé. How to control a biological switch: a mathematical framework for the control of piecewise affine models of gene networks. Research Report 5979, INRIA, 092006.

9. E. Farcot and J.-L. Gouzé. Periodic solutions of piecewise affine gene network models: the case of a negative feedback loop. Research Report 6018, INRIA, 112006.

10. A. F. Filippov. Differential Equations with Discontinuous Righthand Sides. Kluwer Academic Publishers, Dordrecht, 1988.

11. R. Ghosh and C. Tomlin. Symbolic reachable set computation of piecewise affine hybrid automata and its application to biological modelling: Delta-Notch protein signalling. Systems Biology, 1(1):170-183, 2004.

12. L. Glass and S. Kauffman. The logical analysis of continuous non-linear biochemical control networks. J. Theor. Biol., 39:103-129, 1973.

13. J. Gouzé and T. Sari. A class of piecewise linear differential equations arising in biological models. Dyn. Syst, 17:299-316, 2002.

14. L. Habets and J. van Schuppen. A control problem for affine dynamical systems on a full-dimensional polytope. Automatica, 40:21 - 35, 2004.

15. H. M. Hardin and J. van Schuppen. System reduction of nonlinear positive systems by linearization and truncation. In C. Commault and N. Marchand, editors, Positive systems - Proceedings of the Second Multidisciplinary Symposium on Positive Systems: Theory and Applications (POSTA 06), volume 341 of Lecture Notes in Control and Information Sciences, pages 431-438. Grenoble, France, 2006.

16. J. Harman. Allosteric regulation of the cAMP receptor protein. Biochimica et Biophysica Acta, 1547(1):1-17, 2001.

17. K. Heidtke and S. Schulze-Kremer. Design and implementation of a qualitative simulation model of $\lambda$ phage infection. Bioinformatics, 14(1):81-91, 1998.

18. R. Hengge-Aronis. The general stress response in Escherichia coli. In G. Storz and R. Hengge-Aronis, editors, Bacterial Stress Responses, pages 161-177. ASM Press, Washington, DC, 2000.

19. G. Huisman, D. Siegele, M. Zambrano, and R. Kolter. Morphological and physiological changes during stationary phase. In F. Neidhardt, R. Curtiss III, J. Ingraham, E. Lin, K. Low, B. Magasanik, W. Reznikoff, M. Riley, M. Schaechter, and H. Umbarger, editors, Escherichia coli and Salmonella: Cellular and Molecular Biology, pages 1672-1682. ASM Press, Washington, DC, 2nd edition, 1996.

20. S. Kauffman. The Origins of Order: Self-Organization and Selection in Evolution. Oxford University Press, New York, 1993.

21. A. Keil and J.-L. Gouzé. Model reduction of modular systems using balancing methods. Technical report, Munich University of Technology, 2003.

22. I. Koch, B. Junker, and M. Heiner. Application of Petri net theory for modelling and validation of the sucrose breakdown pathway in the potato tuber. Bioinformatics, 2005. In press.

23. K. Kohn. Molecular interaction maps as information organizers and simulation guides. Chaos, 11(1):84-97, 2001.

24. A. Martinez-Antonio and J. Collado-Vides. Identifying global regulators in transcriptional regulatory networks in bacteria. Current Opinion in Microbiology, 6(5):482-489, 2003. 
25. T. Mestl, E. Plahte, and S. Omholt. A mathematical framework for describing and analysing gene regulatory networks. Journal of Theoretical Biology, 176(2):291-300, 1995.

26. M. Ptashne. A Genetic Switch: Phage $\lambda$ and Higher Organisms. Cell Press \& Blackwell Science, Cambridge, MA, 2nd edition, 1992.

27. V. Reddy, M. Liebman, and M. Mavrovouniotis. Qualitative analysis of biochemical reaction systems. Computers in Biology and Medicine, 26(1):9-24, 1996.

28. A. Regev, W. Silverman, and E. Shapiro. Representation and simulation of biochemical processes using the $\pi$-calculus process algebra. In R. Altman, A. Dunker, L. Hunter, K. Lauderdale, and T. Klein, editors, Pacific Symposium on Biocomputing, PSB'01, volume 6, pages 459-470, Singapore, 2001. World Scientific Publishing.

29. D. Ropers, H. de Jong, M. Page, D. Schneider, and J. Geiselmann. Qualitative simulation of the carbon starvation response in Escherichia coli. BioSystems, 84:124-152, 2006.

30. R. Thomas and R. d'Ari. Biological Feedback. CRC Press, Boca Raton, FL, 1990.

31. J. Wang, E. Gilles, J. Lengeler, and K. Jahreis. Modeling of inducer exclusion and catabolite repression based on a PTS-dependent sucrose and non-PTS-dependent glycerol transport systems in Escherichia coli K-12 and its experimental verification. Journal of Biotechnology, 92(2):133-158, 2001.

32. L. Wick and T. Egli. Molecular components of physiological stress responses in Escherichia coli. Advances in Biochemical Engineering/Biotechnology, 89:1-45, 2004. 


\section{Index}

asymptotic dynamics, 13, 16

Filippov, 2, 6-8, 18

focal point, 5, 6, 8, 12

focal set, 6,7

genetic regulatory network, 1

piecewise-linear model, 1 qualitative analysis, 2, 8, 10-12, 21

regulatory domain, 4-7, 9

sliding mode, $3,6,13,18$

strongly connected component, 21

$\operatorname{supp}(\mathrm{D}), 4,6$

switching domain, 4-9

transition graph, 8-10, 12, 13 